\title{
QUADRATICALLY CONVERGING ITERATIVE SCHEMES FOR NONLINEAR VOLTERRA INTEGRAL EQUATIONS AND AN APPLICATION
}

\author{
SUDHAKAR G. PANDIT \\ Winston-Salem State University, Department of Mathematics \\ Winston-Salem, NC 27110 USA
}

(Received March, 1996; Revised September, 1996)

\begin{abstract}
A generalized quasilinear technique is employed to derive iterative schemes for nonlinear Volterra integral equations under various monotonicity and convexity (concavity) conditions on the kernels. The iterates in the schemes are linear, and converge monotonically, uniformly and quadratically to the unique solution. An application to a boundary-layer theory problem and examples illustrating the results are presented.

Key words: Volterra Integral Equations, Generalized Quasilinear Technique, Monotone Iterative Technique, Successive Approximations Technique, Quadratic Convergence, Boundary-Layer Theory.
\end{abstract}

AMS subject classifications: 45G10, 45L10, 34C50.

\section{Introduction}

For the nonlinear Volterra integral equation

$$
u(t)=h(t)+\int_{0}^{t} K(t, s, u(s)) d s
$$

when $K$ is nondecreasing in $u$ and satisfies a Lipschitz condition, the successive approximations method [9] yields a monotonic sequence converging uniformly to the unique solution of (1.1). On the other hand, if $K$ is nonincreasing in $u$ and satisfies a Lipschitz condition, then there is an alternating sequence of successive approximations converging to the unique solution of (1.1) in a closed set bounded by lower and upper functions [8]. The iterates defining the above sequences are nonlinear and the rate of convergence is linear. The iterates employed in the monotone iterative technique [3, 5] are linear, and so is their convergence rate. Based on the quaslinearization idea [1], the generalized quasilinear technique initiated in [6] and later extended in [4] offers two monotonic sequences of linear iterates converging uniformly and quadratically (and hence more rapidly) to the unique solution of the initial value problem

$$
u^{\prime}=f(t, u), \quad u=u(t)
$$

Printed in the U.S.A. (C)1997 by North Atlantic Science Publishing Company 
The generalized quasilinear technique has recently found its way [2] into the initialboundary value problem for the two dimensional analog of (1.2), namely

$$
u_{x y}=f\left(x, y, u, u_{x}, u_{y}\right), \quad u=u(x, y)
$$

whereas it is still open for the (more difficult) periodic-boundary value problem associated with (1.3).

The purpose of this paper is to develop linearly defined and quadratically convergent iterative schemes for (1.1) under various monotonicity and convexity (concavity) conditions on $K$. Of special interest is the case when $K$ is nonincreasing and convex in $u$. The boundary-layer theory problem [10], when transformed into a Volterra integral equation has a nonincreasing and convex kernel (see Example 4.2). It is apt to note that the method of successive approximations when applied to nonlinear problems is especially useful, even for numerical computations, when the nonlinearities are nonincreasing [7, 8]. In this case, we employ coupled lower, and upper solutions in the development of our schemes (see Theorem 3.2). We present examples illustrating the results obtained.

\section{Volterra Integral Inequalities}

For $T \in \mathbb{R}$ and $T>0$, let $J=[0, T]$ and $D=\{(t, s) \in J \times J ; s \leq t\}$. Consider

$$
u(t)=h(t)+\int_{0}^{t} K(t, s, u(s)) d s, \quad t \in J
$$

where $h \in C[J, \mathbb{R}]$ and $K \in C[D \times \mathbb{R}, \mathbb{R}]$.

A function $v \in C[J, \mathbb{R}]$ is called an upper solution of $(2.1)$ on $J$ if

$$
v(t) \geq h(t)+\int_{0}^{t} K(t, s, v(s)) d s, \quad t \in J
$$

and a lower solution, if the reversed inequalities hold. If

$$
v(t) \leq h(t)+\int_{0}^{t} K(t, s, w(s)) d s, w(t) \geq h(t)+\int_{0}^{t} K(t, s, v(s)) d s, \quad t \in J
$$

then $v$ and $w$ are said to be coupled lower and upper solutions of (2.1) on J.

Theorem 2.1: Suppose that

(i) $\quad K_{1}, K_{2} \in C[D \times \mathbb{R}, \mathbb{R}] ; h, v, w \in C[J, \mathbb{R}]$, and the inequalities

$$
\begin{aligned}
& v(t) \leq h(t)+\int_{0}^{t} K_{1}(t, s, v(s)) d s+\int_{0}^{t} K_{2}(t, s, w(s)) d s, \\
& w(t) \geq h(t)+\int_{0}^{t} K_{1}(t, s, w(s)) d s+\int_{0}^{t} K_{2}(t, s, v(s)) d s
\end{aligned}
$$

hold for $t \in J$; 
(ii) $\quad K_{1}$ is nondecreasing and $K_{2}$ is nonincreasing in $u$ for each fixed $(t, s) \in D$;

(iii) $\quad K_{1}$ and $K_{2}$ satisfy one-sided Lipschitz conditions

$$
K_{1}(t, s, \alpha)-K_{1}(t, s, \beta) \leq L(\alpha-\beta), \quad K_{2}(t, s, \alpha)-K_{2}(t, s, \beta) \geq-L(\alpha-\beta),
$$

whenever $\alpha \geq \beta$ and $L>0$ is a constant.

Then, $v(0) \leq w(0)$ implies

$$
v(t) \leq w(t), \quad 0<t \leq T
$$

Proof: For $\epsilon>0$, sufficiently small, set $v_{\epsilon}(t)=v(t)-\epsilon \exp (2 L T), w_{\epsilon}(t)=w(t)+$ $\epsilon \exp (2 L t)$, so that $v_{\epsilon}<v$ and $w_{\epsilon}>w$ on $J$, and from conditions (i)-(iii) we obtain

and

$$
v_{\epsilon}(t)<h(t)+\int_{0}^{t}\left[K_{1}\left(t, s, v_{\epsilon}(s)\right)+K_{2}\left(t, s, w_{\epsilon}(s)\right)\right] d s
$$

$$
w_{\epsilon}(t)>h(t)+\int_{0}^{t}\left[K_{1}\left(t, s, w_{\epsilon}(s)\right)+K_{2}\left(t, s, v_{\epsilon}(s)\right)\right] d s .
$$

Therefore, it suffices to show that

$$
v_{\epsilon}(t)<w_{\epsilon}(t), \text { for } 0<t \leq T \text {. }
$$

To this end, suppose that there is a point $t_{0}, 0<t_{0} \leq T$, such that $v_{\epsilon}(\tau)<w_{\epsilon}(\tau)$ for $0<\tau<t_{0}$ and $v_{\epsilon}\left(t_{0}\right)=w_{\epsilon}\left(t_{0}\right)$. Then conditions $(i)$ and (ii) yield the contradiction

$$
\begin{gathered}
v_{\epsilon}\left(t_{0}\right)<h\left(t_{0}\right)+\int_{0}^{t_{0}}\left[K_{1}\left(t_{0}, s, v_{\epsilon}(s)\right)+K_{2}\left(t_{0}, s, w_{\epsilon}(s)\right)\right] d s \\
\leq h\left(t_{0}\right)+\int_{0}^{t_{0}}\left[K_{1}\left(t_{0}, s, w_{\epsilon}(s)\right)+K_{2}\left(t_{0}, s, v_{\epsilon}(s)\right)\right] d s \\
<w_{\epsilon}\left(t_{0}\right),
\end{gathered}
$$

which justifies (2.3) and therewith the theorem.

Utilizing the ideas of Theorem 2.1 we can prove the existence of solutions of a finite system of Volterra integral equations in a closed set. (See [3] for a similar result for systems of ordinary differential equations.) In what follows, vectorial inequalities mean that the same inequalities hold for their corresponding components. Recall that a vector function is continuous or nondecreasing (nonincreasing) when all its components are such. For $v_{0}, w_{0} \in C\left[J, \mathbb{R}^{n}\right]$, such that $v_{0}(t) \leq w_{0}(t)$ on $J$, let $\Omega_{n}=\left\{(t, s, u) \in D \times \mathbb{R}^{n}: v_{0}(t) \leq u \leq w_{0}(t)\right\}$. Consider the system

$$
u(t)=h(t)+\int_{0}^{t} R(t, s, u(s)) d s, \quad t \in J
$$


Theorem 2.2: Let $u, h \in C\left[J, \mathbb{R}^{n}\right]$ and $R(t, s, u) \in C\left[\Omega_{n}, \mathbb{R}^{n}\right]$.

(a) If $v_{0}, w_{0} \in C\left[J, \mathbb{R}^{n}\right], v_{0}(t) \leq w_{0}(t)$ on $J$, are lower and upper solutions of (2.4) on $J$ respectively, and $R$ is nondecreasing in $u$ for each fixed pair of $s$ and $t$, then there exists a solution $u$ of (2.4) such that

$$
v_{0}(t) \leq u(t) \leq w_{0}(t) \text { on } J
$$

Furthermore, if $R$ satisfies Lipschitz condition

$$
\left|R_{j}(t, s, u)-R_{j}(t, s, v)\right| \leq L_{j} \sum_{i=1}^{n}\left|u_{i}-v_{i}\right|, \quad 1 \leq j \leq n,
$$

then, $u$ is unique;

(b) If $v_{0}, w_{0} \in C\left[J, \mathbb{R}^{n}\right], v_{0}(t) \leq w_{0}(t)$ on $J$, are coupled lower and upper solutions of (2.4) on $J$ and $R$ is nonincreasing in $u$ for each fixed pair of $s$ and $t$, then there exists a solution (unique solution, if $R$ satisfies (2.6)) $u$ of (2.4) satisfying (2.5).

The following vector integral inequality of Gronwall type is required in establishing the quadratic convergence of iterates.

Lemma 2.1: Let $u(t) \leq h(t)+\int_{0}^{t} A u(s) d s, \quad t \in J$ where $u, h \in C\left[J, \mathbb{R}^{n}\right]$ and $A=\left(a_{i j}\right)$ is an $n \times n$ constant matrix with $a_{i j} \geq 0$. Then,

$$
u(t) \leq h(t)+\int_{0}^{t} A \exp (A(t-s)) h(s) d s, \quad t \in J .
$$

\section{Generalized Quasilinearization}

In the result below, we employ upper and lower solutions to develop a quadratically convergent iterative scheme for (2.1) when the kernel $K$ is nondecreasing and convex in $u$. For $v_{0}, w_{0} \in C[J, \mathbb{R}]$ and $v_{0}(t) \leq u \leq w_{0}(t)$ on $J$, let $\Omega=\{(t, s, u) \in D \times \mathbb{R}$; $\left.v_{0}(t) \leq u \leq w_{0}(t), t \in J\right\}$. Let $\|u\|=\max |u(t)|, t \in J$.

Theorem 3.1: Suppose that

(i) $\quad v_{0}, w_{0} \in C[J, \mathbb{R}], v_{0}(t) \leq w_{0}(t)$ on $J$, and $v_{0}, w_{0}$ are lower and upper solutions of (2.1) on $J$, respectively;

(ii) $K \in C^{2}[\Omega, \mathbb{R}], K_{u}(t, s, u) \geq 0, K_{u u}(t, s, u) \geq 0$, for $(t, s, u) \in \Omega$.

Then, there exist a nondecreasing sequence $\left\{v_{n}(t)\right\}$ and a nonincreasing sequence $\left\{w_{n}(t)\right\}$ in $C[J, \mathbb{R}]$ such that $v_{n} \rightarrow u$ and $w_{n} \rightarrow u$ uniformly on $J$, and the following quadratic convergence estimates hold:

$$
\begin{gathered}
\left\|u-v_{n}\right\| \leq A\left\|u-v_{n-1}\right\|^{2} \\
\left\|w_{n}-u\right\| \leq B\left\|w_{n-1}-u\right\|^{2}+A\left\|u-v_{n-1}\right\|^{2}
\end{gathered}
$$

for $n=1,2, \ldots$, where $A>0$ and $B>0$ are constants.

Proof: By assumption (ii), we have

and

$$
K\left(t, s, u_{2}\right) \geq K\left(t, s, u_{1}\right)+K_{u}\left(t, s, u_{1}\right)\left(u_{2}-u_{1}\right),
$$




$$
K\left(t, s, u_{2}\right)-K\left(t, s, u_{1}\right) \leq L\left(u_{2}-u_{1}\right)
$$

for $\left(t, s, u_{1}\right),\left(t, s, u_{2}\right) \in \Omega, u_{2} \geq u_{1}$, and $L>0$ a constant. Consider the linear Volterra integral equations

$$
v(t)=h(t)+\int_{0}^{t} K_{1}\left(t, s, v_{0}(s) ; v(s)\right) d s, t \in J,
$$

and

$$
w(t)=h(t)+\int_{0}^{t} K_{2}\left(t, s, v_{0}(s), w_{0}(s) ; w(s)\right) d s, t \in J
$$

where

$$
K_{1}\left(t, s, v_{0}(s) ; v(s)\right)=K\left(t, s, v_{0}(s)\right)+K_{u}\left(t, s, v_{0}(s)\right)\left(v(s)-v_{0}(s)\right)
$$

and

$$
K_{2}\left(t, s, v_{0}(s), w_{0}(s) ; w(s)\right)=K\left(t, s, w_{0}(s)\right)+K_{u}\left(t, s, v_{0}(s)\right)\left(w(s)-w_{0}(s)\right)
$$

From (3.2), it follows that $v_{0}, w_{0}$ are lower and upper solutions of (3.4) and of (3.4) respectively. Since $K_{u} \geq 0$, and $K_{1}$ and $K_{2}$ are nondecreasing in $v$ and $w$, respectively, by Theorem $2.2(a)$ and the fact that $(3.4)$ and $(3.4)^{\prime}$ are linear, there exist a unique solution $v_{1}(t)$ of $(3.4)$ and a unique solution $w_{1}(t)$ of $(3.4)^{\prime}$ such that $v_{0}(t) \leq$ $v_{1}(t) \leq w_{0}(t)$ and $v_{0}(t) \leq w_{1}(t) \leq w_{0}(t)$ on $J$. Now,

$$
\begin{gathered}
v_{1}(t)=h(t)+\int_{0}^{t}\left[K\left(t, s, v_{0}(s)\right)+K_{u}\left(t, s, v_{0}(s)\right)\left(v_{1}(s)-v_{0}(s)\right)\right] d s \\
\leq h(t)+\int_{0}^{t} K\left(t, s, v_{1}(s)\right) d s,
\end{gathered}
$$

and

$$
\begin{gathered}
w_{1}(t)=h(t)+\int_{0}^{t}\left[K\left(t, s, w_{0}(s)\right)+K_{u}\left(t, s, v_{0}(s)\right)\left(w_{1}(s)-w_{0}(s)\right)\right] d s \\
\geq h(t)+\int_{0}^{t}\left\{K t, s, w_{1}(s)\right)+\left[K_{u}\left(t, s, w_{1}(s)-K_{u}\left(t, s, v_{0}(s)\right)\right]\left(w_{0}(s)-w_{1}(s)\right)\right\} d s \\
\geq h(t)+\int_{0}^{t} K\left(t, s, w_{1}(s)\right) d s .
\end{gathered}
$$

To arrive at (3.5) and $(3.5)^{\prime}$, we have used (3.2) and the facts that $v_{1}(t) \geq v_{0}(t)$, $w_{0}(t) \geq w_{1}(t), \quad w_{1}(t) \geq v_{0}(t)$ on $J$, and $K_{u}(t, s, u)$ is nondecreasing in $u$. Also, $v_{1}(0)=h(0)=w_{1}(0), K$ nondecreasing in $u$ and satisfies one-sided Lipschitz condition (3.3). Therefore, Theorem 2.1 implies that $v_{1}(t) \leq w_{1}(t)$ on $J$, and, consequently, we have $v_{0}(t) \leq v_{1}(t) \leq w_{1}(t) \leq w_{0}(t)$ on $J$. Continuing this process, we obtain a nondecreasing sequence $\left\{v_{n}(t)\right\}$ and a nonincreasing sequence $\left\{w_{n}(t)\right\}$ in $C[J, \mathbb{R}]$ where $v_{n}(t)$ and $w_{n}(t)$ are (unique) solutions of the linear Volterra integral equations 
and

$$
v_{n}(t)=h(t)+\int_{0}^{t} K_{1}\left(t, s, v_{n-1}(s) ; v_{n}(s)\right) d s, t \in J
$$

respectively, and satisfy

$$
w_{n}(t)=h(t)+\int_{0}^{t} K_{2}\left(t, s, v_{n-1}(s), w_{n-1}(s) ; w_{n}(s)\right) d s, t \in J,
$$

$$
v_{0}(t) \leq v_{1}(t) \leq v_{2}(t) \leq \ldots \leq v_{n}(t) \leq w_{n}(t) \leq \ldots \leq w_{2}(t) \leq w_{1}(t) \leq w_{0}(t)
$$

on $J$. Employing standard arguments [3], it is easy to see that $v_{n} \rightarrow u$ and $w_{n} \rightarrow u$ uniformly on $J$, where $u$ is the unique solution of (2.1) on $J$. It remains to show that the sequences satisfy the estimates (3.1). To this end, by setting $p_{n}(t)=$ $u(t)-v_{n}(t) \geq 0$, and $q_{n}(t)=w_{n}(t)-u(t) \geq 0$, we obtain

$p_{n}(t)$

$$
\begin{gathered}
=\int_{0}^{t}\left[K(t, s, u(s))-K\left(t, s, v_{n-1}(s)\right)-K_{u}\left(t, s, v_{n-1}(s)\right)\left(p_{n-1}(s)-p_{n}(s)\right)\right] d s \\
\leq \int_{0}^{t} K_{u u}(t, s, \alpha(s))\left[p_{n-1}(s)\right]^{2} d s+\int_{0}^{t} K_{u}\left(t, s, v_{n-1}(s)\right) p_{n}(s) d s \\
\leq \int_{0}^{t} M_{1}\left[p_{n-1}(s)\right]^{2} d s+\int_{0}^{t} M_{2} p_{n}(s) d s
\end{gathered}
$$

where $v_{n-1}<\alpha<u, M_{1}=\max K_{u u}$, and $M_{2}=\max K_{u}$. Similarly,

$q_{n}(t)$

$$
\begin{gathered}
=\int_{0}^{t}\left[K t, s, w_{n-1}(s)-K(t, s, u(s))+K_{u}\left(t, s, v_{n-1}(s)\right)\left(q_{n}(s)-q_{n-1}(s)\right)\right] d s \\
\leq \int_{0}^{t} K_{u u}(t, s, \beta(s))\left[q_{n-1}(s)+p_{n-1}(s)\right) q_{n-1}(s) d s \\
\quad+\int_{0}^{t} K_{u}\left(t, s, v_{n-1}(s)\right) q_{n}(s) d s \\
\leq \int_{0}^{t}\left\{2 M_{1}\left[q_{n-1}(s)\right]^{2}+M_{1}\left[p_{n-1}(s)\right]^{2}\right\} d s+\int_{0}^{t} M_{2} q_{n}(s) d s
\end{gathered}
$$

where $v_{n-1}<\beta<w_{n-1}$. The desired inequalities in (3.1) now follow by applying Lemma 2.1 to (3.7) and (3.7)', thereby completing the proof.

In our next result, we employ coupled lower and upper solutions to make use of the predominant effect of the nonincreasing character of $K$.

Theorem 3.2: Suppose that 
(i) $\quad v_{0}, w_{0} \in C[J, \mathbb{R}], v_{0}(t) \leq w_{0}(t)$ on $J$, and $v_{0}, w_{0}$ are coupled lower and upper solutions of $(2.1)$ on $J$;

(ii) $\quad K \in C^{2}[\Omega, \mathbb{R}], K_{u}(t, s, u) \leq 0, K_{u u}(t, s, u) \geq 0$, for $(t, s, u) \in \Omega$.

Then, there exist monotone sequences $\left\{v_{n}(t)\right\}$ and $\left\{w_{n}(t)\right\}$ converging uniformly and quadratically to the unique solution of (2.1) on $J$.

Proof: The assumption that $K_{u u} \geq 0$ yields the following inequalities which, in view of the fact that $K_{u} \leq 0$, are better suited in the context of coupled lower and upper solutions:

and

$$
K\left(t, s, u_{2}\right) \leq K\left(t, s, u_{1}\right)+K_{u}\left(t, s, u_{2}\right)\left(u_{2}-u_{1}\right)
$$

$$
K\left(t, s, u_{2}\right)-K\left(t, s, u_{1}\right) \geq-L\left(u_{2}-u_{1}\right)
$$

for $\left(t, s, u_{1}\right),\left(t, s, u_{2}\right) \in \Omega, u_{2} \geq u_{1}$, and $L>0$, a constant. For $n=1,2, \ldots$, consider the coupled system of linear Volterra integral equations

$$
v_{n}(t)=h(t)+\int_{0}^{t} R_{1}\left(t, s, w_{n-1}(s) ; w_{n}(s)\right) d s, t \in J
$$

and

where

$$
w_{n}(t)=h(t)+\int_{0}^{t} R_{2}\left(t, s, w_{n-1}(s), v_{n-1}(s) ; v_{n}(s)\right) d s, t \in J
$$

$$
\begin{gathered}
R_{1}\left(t, s, w_{n-1}(s) ; w_{n}(s)\right) \\
=K\left(t, s, w_{n-1}(s)\right)+K_{u}\left(t, s, w_{n-1}(s)\right)\left[w_{n}(s)-w_{n-1}(s)\right]
\end{gathered}
$$

and

$$
\begin{gathered}
R_{2}\left(t, s, w_{n-1}(s), v_{n-1}(s) ; v_{n}(s)\right)=K\left(t, s, v_{n-1}(s)\right) \\
+K_{u}\left(t, s, w_{n-1}(s)\right)\left[v_{n}(s)-v_{n-1}(s)\right] .
\end{gathered}
$$

As in Theorem 3.1, we obtain two monotone sequences $\left\{v_{n}\right\}$ and $\left\{w_{n}\right\}$ satisfying (3.6) and converging uniformly to the unique solution $u$ of $(2.1)$ on $J$. Setting $p_{n}(t)=u(t)-v_{n}(t)$ and $q_{n}(t)=w_{n}(t)-u(t)$ we obtain the following inequalities in place of (3.7) and (3.7)':

and

$$
p_{n}(t) \leq \int_{0}^{t} M_{1}\left[q_{n-1}(s)\right]^{2} d s+\int_{0}^{t} M_{2} q_{n}(s) d s
$$

$$
q_{n}(t) \leq \int_{0}^{t}\left\{2 M_{1}\left[p_{n-1}(s)\right]^{2} d s+M_{1}\left[q_{n-1}(s)\right]^{2}\right\} d s+\int_{0}^{t} M_{2} p_{n}(s) d s
$$

with the same constants $M_{1}$ and $M_{2}$ as before. Let

$$
r_{n}(t)=\left[\begin{array}{c}
p_{n}(t) \\
q_{n}(t)
\end{array}\right], \quad P=\left[\begin{array}{cc}
0 & M_{2} \\
M_{2} & 0
\end{array}\right], \text { and } Q=\left[\begin{array}{cc}
0 & M_{1} \\
2 M_{1} & M_{1}
\end{array}\right] .
$$


Then $(3.10)$ and $(3.10)^{\prime}$ can be written as a system

$$
r_{n}(t) \leq \int_{0}^{t} Q\left[r_{n-1}(s)\right]^{2} d s+\int_{0}^{t} \operatorname{Pr}_{n}(s) d s .
$$

Lemma 2.1 now yields the required quadratic estimate

$$
\left\|r_{n}\right\| \leq Q \exp (A T) T\left\|r_{n-1}\right\|^{2}
$$

and the proof is complete.

\section{Examples and Other Related Results}

The following examples illustrate some of the results in Section 3.

Example 4.1: Consider

where

$$
u(t)=\int_{0}^{t} K(t, s, u(s)) d s
$$

$$
K(t, s, u)=\left\{\begin{array}{cc}
s t-2, & \text { if } u<0 \\
s t-2 \cos u, & \text { if } 0 \leq u \leq \frac{\pi}{2} \\
s t, & \text { if } u>\frac{\pi}{2} .
\end{array}\right.
$$

Then, $K$ is nondecreasing and convex on $[0,1] \times[0,1] \times\left[0, \frac{\pi}{2}\right]$ and $v_{0}(t)=-2 t$ and $w_{0}(t)=t$ are lower and upper solutions of $(4.1)$ on $[0,1]$ respectively. Theorem 3.1 therefore applies.

Example 4.2: The third order ordinary differential equation

$$
y^{\prime \prime \prime}+2 y y^{\prime \prime}=0, \quad 0 \leq t<\infty, y(0)=y^{\prime}(0)=0, y^{\prime \prime}(0)=1
$$

arises in connection with the boundary-layer theory of fluid flow [10]. The transformation $u=-\ln y^{\prime \prime}$ reduces (4.3) to the Volterra integral equation

in which the kernel

$$
u(t)=\int_{0}^{t} K(t, s, u(s)) d s,
$$

$$
K(t, s, u)=\left\{\begin{array}{cc}
(t-s)^{2} \exp (-u), & \text { if } u \geq 0 \\
(t-s)^{2}, & \text { if } u<0
\end{array}\right.
$$

is nonincreasing and convex in $u$. The functions $v_{0}(t) \equiv 0$ and $w_{0}=\frac{t^{3}}{3}$ form a pair of coupled lower and upper solutions of (4.4) on any interval $J=[0, T], T>0$. Theorem 3.2 therefore yields a quadratically convergent linear iterative scheme for (4.4) whose convergence is more rapid than the (nonlinear) alternating sequence scheme of [8, page 282].

By appropriately modifying kernel inequalities (3.2), (3.3), (3.8), (3.9), and defining the iterates $v_{n}$ and $w_{n}$ accordingly, we can obtain the following related results. 
Theorem 4.1: Theorem 3.1 holds if condition(ii) therein is replaced by

$(i i)^{\prime} \quad K \in C^{2}[\Omega, \mathbb{R}], K_{u}(t, s, u) \geq 0, K_{u u}(t, s, u) \leq 0$, for $(t, s, u) \in \Omega$.

Theorem 4.2: Theorem 3.2 holds if condition (ii) therein is replaced by

(ii) $)^{\prime \prime} \quad K \in C^{2}[\Omega, \mathbb{R}], K_{u}(t, s, u) \leq 0, K_{u u}(t, s, u) \leq 0$, for $(t, s, u) \in \Omega$.

Example 4.3: Let $A>0$ and $0<\alpha<1$ be real numbers. The kernel

$$
K(t, s, u)=\left\{\begin{array}{cc}
0, & \text { if } u<0 \\
(u+1)^{\alpha}, & \text { if } 0 \leq u \leq A \\
(A+1)^{\alpha}, & \text { if } u>A
\end{array}\right.
$$

satisfies the conditions of Theorem 4.1 with $h(t) \equiv 0, v_{0}(t)=t$, and $w_{0}(t)=$ $(A+1)^{\alpha} t$. The kernel

$$
K(t, s, u)=\left\{\begin{array}{cc}
0, & \text { if } u<0 \\
-u^{2}, & \text { if } 0 \leq u \leq A \\
-A^{2}, & \text { if } u>A
\end{array}\right.
$$

satisfies the conditions of Theorem 4.2 , with $v_{0}=-A^{2} t$ and $w_{0}(t) \equiv 0$ as a pair of coupled upper and lower solutions, and $h(t) \equiv 0$.

Example 4.4: Consider

$$
u(t)=\int_{0}^{t} K(t, s, u(s)) d s
$$

where

$$
K(t, s, u)=\left\{\begin{array}{cc}
s t-2, & u<0 \\
s t-2 \cos u-u^{2}, & 0 \leq u \leq \frac{\pi}{2} \\
s t-\frac{\pi^{2}}{4}, & u>\frac{\pi}{2} .
\end{array}\right.
$$

Notice that $K$ in (4.8) is not of any of the types considered in Examples 4.1-4.3 but can be expressed as $K=K_{1}+K_{2}$ where $K_{1}$ is of type (4.2) and $K_{2}$ is of type (4.6). The following result, which combines the features of Theorem 3.1 and Theorem 4.2, is applicable to (4.7).

Theorem 4.3: Let $K=K_{1}+K_{2}$. Suppose that

$$
\begin{aligned}
h, v_{0}, w_{0} \in C[J, \mathbb{R}], v_{0} \leq w_{0} \text { on } J, \text { and satisfy } \\
v_{0}(t) \leq h(t)+\int_{0}^{t}\left[K_{1}\left(t, s, v_{0}(s)\right)+K_{2}\left(t, s, w_{0}(s)\right)\right] d s, t \in J, \\
\left.w_{0}(t) \geq h(t)+\int_{0}^{t}\left[K_{1}\left(t, s, w_{0}(s)\right)+K_{2} t, s, v_{0}(s)\right)\right] d s, t \in J
\end{aligned}
$$

$$
\begin{aligned}
& K_{1}, K_{2} \in C[\Omega, \mathbb{R}], K_{1 u}(t, s, u) \geq 0, K_{2 u}(t, s, u) \leq 0, K_{1 u u}(t, s, u) \geq 0 \\
& K_{2 u u}(t, s, u) \leq 0, \text { for }(t, s, u) \in \Omega .
\end{aligned}
$$

Then, there exist monotone sequences $\left\{v_{n}\right\}$ and $\left\{w_{n}\right\}$ on $J$, converging uniformly and quadratically to the unique solution of (4.7) on $\mathrm{J}$. 


\section{References}

[1] Bellman, R. and Kalaba, R.E., Quasilinearization and Nonlinear Boundary Value Problems, Elsevier, New York 1965.

[2] Deo, S.G. and Pandit, S.G., Method of generalized quasilinearization for hyperbolic initial-boundary value problems, Nonlinear World 3 (1996), 267-275.

[3] Ladde, G.S., Lakshmikantham, V. and Vatsala, A.S., Monotone Iterative Techniques for Nonlinear Differential Equations, Pitman, Boston 1985.

[4] Lakshmikantham, V., Leela, S. and Sivasundaram, S., Extensions of the method of quasilinearization, J. Opt. Th. Appl. (to appear).

[5] Ladde, G.S., Lakshmikantham, V. and Pachpatte, B.G., The method of upper and lower solutions and Volterra integral equations, J. Integral Eqs. 4 (1982), 353-360.

[6] Lakshmikantham, V. and Malek, S., Generalized quasilinearization, Nonlinear World 1 (1994), 59-63.

[7] Langer, R.E., (ed.), Boundary Value Problems in Differential Equations The University of Wisconsin Press, Madison Wisconsin 1960.

[8] Saaty, T.L., Modern Nonlinear Equations, Dover Publications, New York 1981.

[9] Walter, W., Differential- und Integralungleichungen, Springer-Verlag OHG, Berlin 1964.

[10] Weil, H., On the differential equations of the simplest boundary-layer theory problems, Ann. of Math. 43 (1942), 381-407. 




Advances in

Operations Research

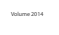

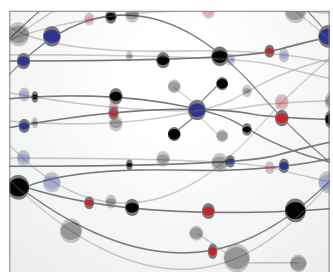

\section{The Scientific} World Journal
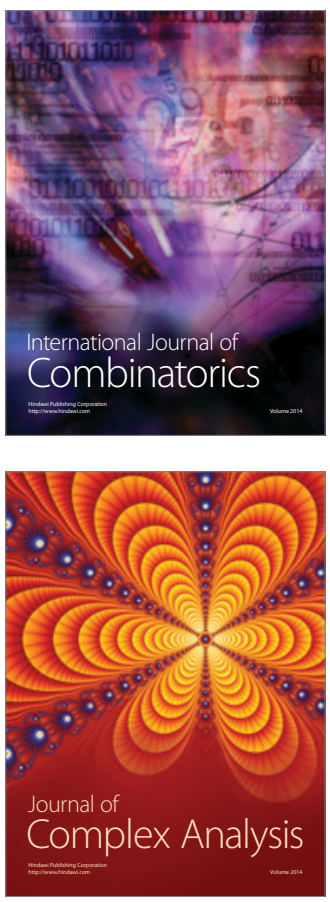

International Journal of

Mathematics and

Mathematical

Sciences
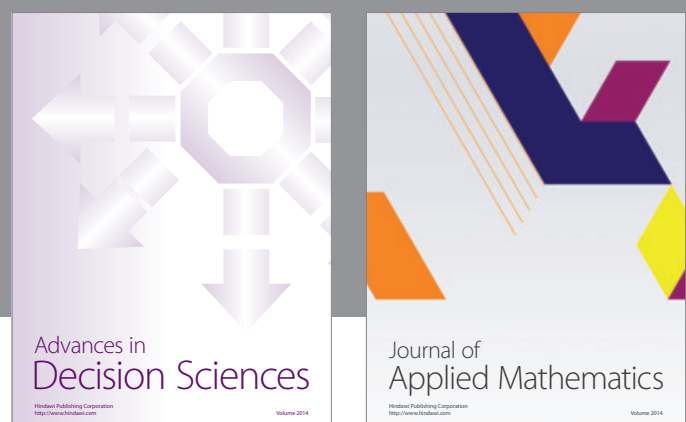

Journal of

Applied Mathematics


Submit your manuscripts at http://www.hindawi.com


Mathematical Problems in Engineering
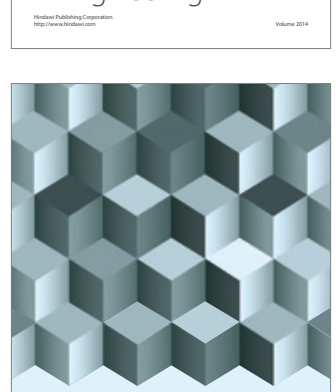

Journal of

Function Spaces
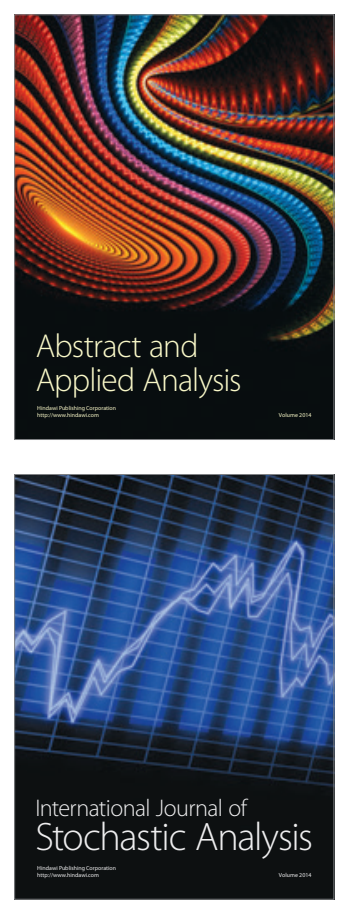

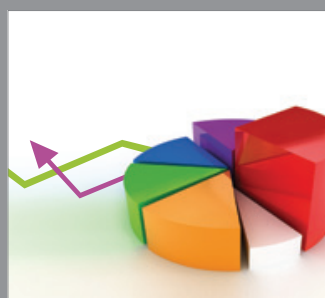

ournal of

Probability and Statistics

Promensencen
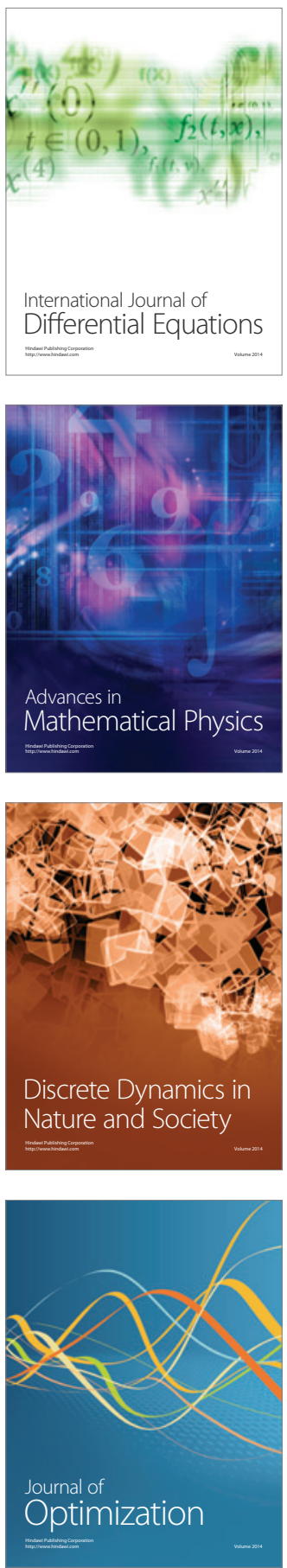